\title{
Active textile antennas as a platform for more energy-efficient and reliable wireless links in healthcare
}

\author{
Hendrik Rogier ${ }^{1,2}$, Frederick Declercq ${ }^{1,2}$ Patrick Van Torre $^{1}$, and Luigi \\ Vallozzi $^{1}$ \\ 1 Ghent University, Dept. of Information Technology \\ Sint-Pietersnieuwstraat 41, B-9000 Ghent, Belgium \\ Hendrik.Rogier@intec.UGent.be, \\ 2 IMEC, INTEC Division, Ghent,Belgium
}

\begin{abstract}
New wireless wearable monitoring systems worn by patients and caregivers require a high degree of reliability and autonomy. We show that active textile antenna systems may serve as robust platforms to deploy such wireless links, in the meanwhile being comfortable and invisible to the wearer, thanks to recent developments in the design process combined with dedicated signal processing techniques. The key idea is to exploit the large amount of real estate available in patients' and caregivers' garments to deploy multiple textile antennas each with a size large enough to make them efficient radiators when deployed on the body. The antenna area is then reused by positioning active electronics directly underneath and energy harvesters directly on top of the antenna patch, ensuring the autonomy of the module. Combining different antenna signals by means of low-power multi-antenna processing techniques then ensures good signal quality at low transmit power in all situations.
\end{abstract}

Key words: Smart Fabrics and Interactive Textiles, wearable electronics, active textile antennas, MIMO signal processing

\section{Introduction}

The explosive developments in the field of smart fabrics and interactive textiles (SFIT) have opened a wide range of exciting possibilities in the field of healthcare. Remote monitoring of patients may be provided by wearable systems that are unobtrusively integrated into patient garments, in the meanwhile continuously monitoring life signs, activities and environmental conditions, and relaying these data wirelessly to a remote location for supervision by a caregiver. Such intelligent garments may be deployed both in the hospital and in the home environment of the wearer. Also caregivers may be equipped with wearable systems, either to collect and transmit their own parameters during interventions in hazardous situations, or as a personal wearable computer that receives, interprets and displays patient data to help a caregiver in making decisions. 
As SFIT systems are to be deployed in critical applications such as patient monitoring and rescue missing, their reliability and autonomy are two key concerns of the designers. To ensure sufficient autonomy without the use of heavy batteries, we need to satisfy two requirements: First, the wireless communication module, and in particular the transceiver module responsible for setting up the wireless link is typically one of the largest energy consumers of the wearable system. Therefore, we need to design a highly energy-efficient communication system, in which textile antennas are critical components. Indeed, an efficient wearable antenna providing off-body communication with high gain and large radiation efficiency typically consumes quite a lot of space, as a large ground plane is required to avoid absorption of antenna radiation by the human body. Yet, this space is widely available in garments. However, the large flexible textile antenna $[6,4,5,3]$ will typically be subject to bending, wrinkling and crumpling as the wearer moves around. This should be taken into account during the design phase of these antennas. Second, to ensure sufficient autonomy to the SFIT system, energy harvesters should be added that make use of the large number of energy sources available in the neighbourhood of the body to scavenge energy.

In this contribution, we will review and evaluate a number of techniques that may be implemented on textile antennas to increase the energy-efficiency and autonomy of SFIT systems. The key idea is to increase performance and functionality by reusing surface area for multiple purposes. This is done at two levels: First, the garment, offering protection and comfort to the wearer, is reused as a platform for a wearable multi-antenna system. Second, the textile antenna itself, being the largest component of each wearable module, is reused as a platform for active electronic circuits implementing sensing and communication, and for energy-scavengers with energy-management circuits.

In Section 2 we show how active electronic circuits may be directly integrated underneath the wearable antenna, resulting in a compact communication module. Moreover, by adopting a full-wave/circuit co-design and co-optimizing strategy, optimal active antenna performance can be obtained, aiming either for optimal noise or impedance matching. Next, we show in Section 3 how one can integrate a set of solar cells on top of the antenna patch, without disturbing the radiation characteristics of the textile antennas. In Section 4 we show that by combining multiple wearable antennas, integrating them in the front and back sections of a garment and relying on diversity techniques at both ends of the link, the reliability of wireless communication links can be improved tremendously. Diversity techniques can easily be implemented at the receiver side, but the use of space-time codes can be quite energy-consuming as codes must be generated at the transmitting side, whereas accurate channel estimation is required at the receiver. Section 5 presents some energy-efficient alternatives, such as passive beamforming and channel state tracking. It is shown that these techniques provide an almost equal performance while relying on much simpler and less energy-consuming signal processing algorithms. Finally, we formulate some conclusions in Section 6. 

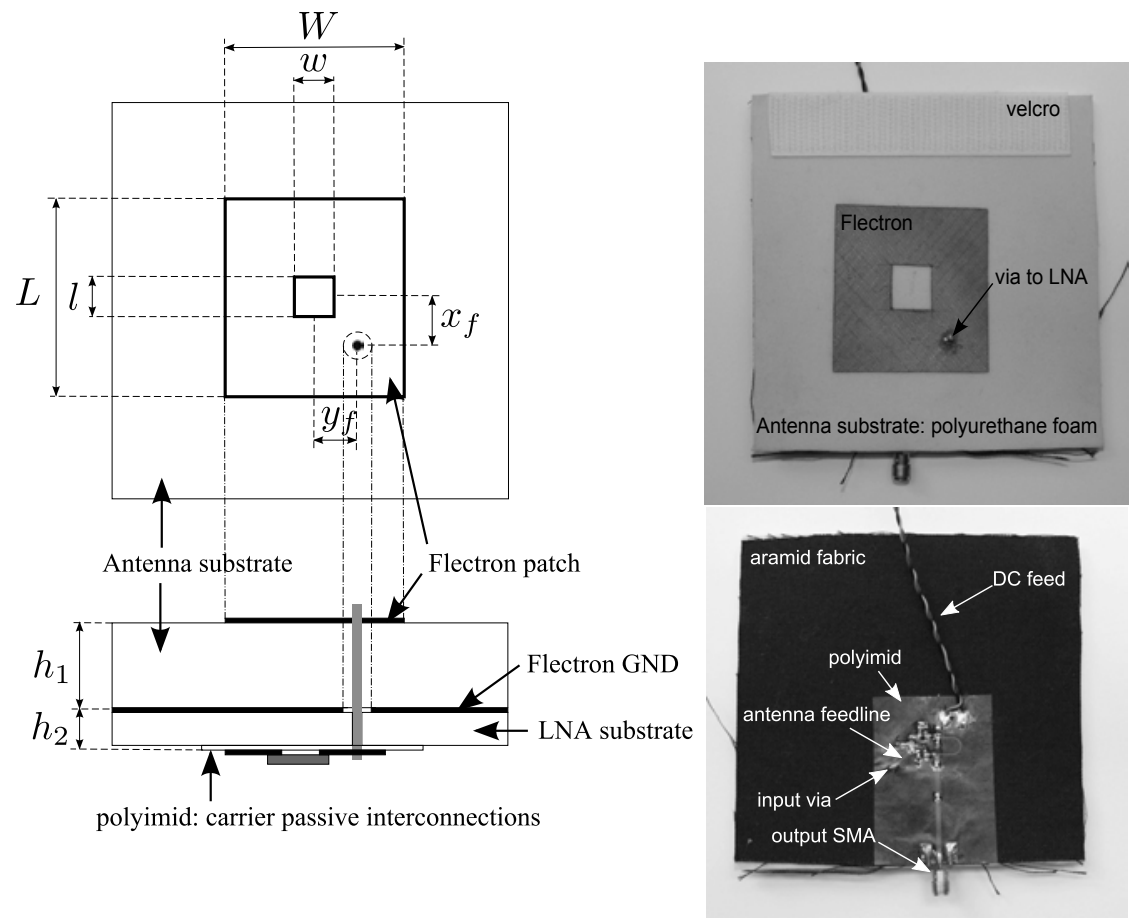

Fig. 1. Active wearable receive antenna operating in $2.45 \mathrm{GHz}$ ISM band.

\section{Full-wave/circuit co-design of active wearable antennas}

Although the development of wearable electronics systems has boomed over the last decade, most of the research focused on converting conventional rigid electronic circuits into flexible wearable components that are compatible with integration into fabric. The complete wearable electronic system is then constructed by interconnecting the set of wearable components needed to implement the desired functionality. This approach, however, results in a fragile overall system, with many weak links that easily break. Moreover, as all components are designed separately, optimal performance of the overall system is not guaranteed. Therefore, we have adopted a new design strategy [2] where we aim to integrate as many components as possible on the textile antenna(s) of the wearable system, in the meanwhile reusing the large area that we need to ensure high gain and good radiation efficiency, and opening new opportunities to co-optimize the performance of the antenna together with the active circuits. At the same time, improving overall system performance also increases the autonomy of the wireless system and reduces the risk of interference with other systems. Both problems are highly relevant in healthcare applications.

In Fig. 1 we present an active wearable antenna, for which the full-wave/circuit co-design approach was applied to integrate a low-noise amplifier (LNA) directly underneath a textile patch antenna. As the antenna feed is directly connected 

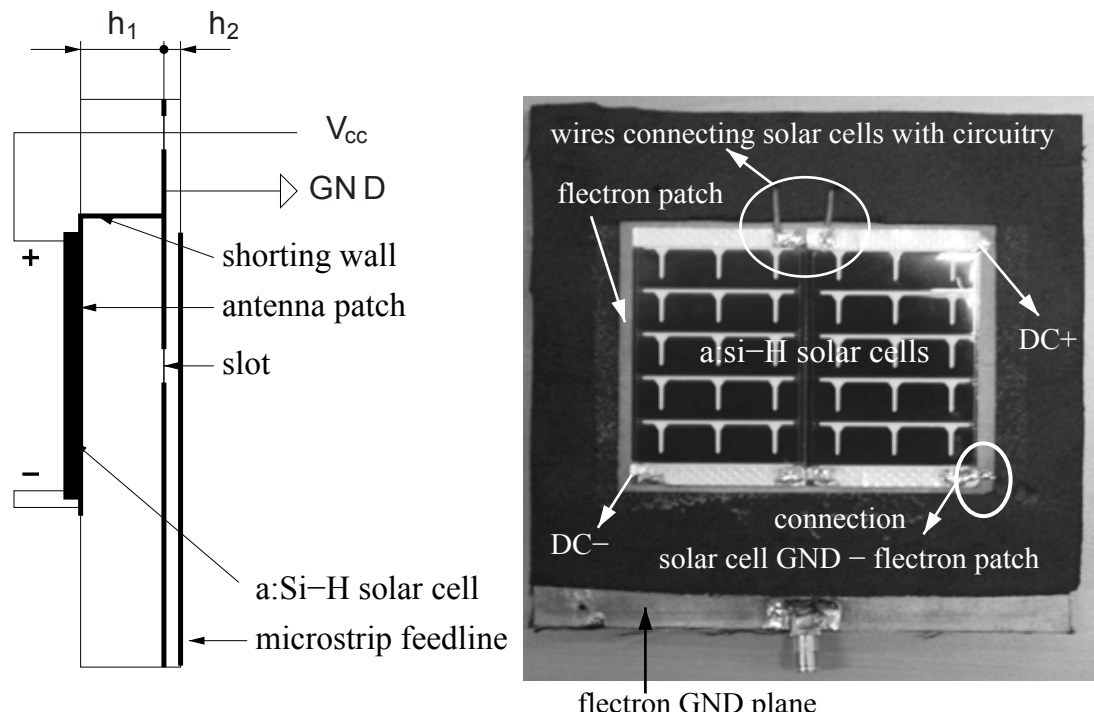

Fig. 2. Aperture-coupled shorted wearable solar patch antenna for 902-928 MHz UHF band.

to the LNA input by means of a short via, RF connection losses are reduced. Moreover, careful co-optimization of the patch dimensions, the position of the antenna feed point and the elements composing the LNA circuit results in optimal noise performance, high gain and $50 \Omega$ matching at the output of the LNA, leading to a highly sensitive receive antenna that operates in the $2.45 \mathrm{GHz}$ ISM band.

\section{Integration of a solar cell on a wearable antenna}

Ensuring acceptable autonomy is of major concern for wearable systems, since batteries rapidly increase the weight and may reduce the flexibility. An exciting new area of the research focusses on harvesting energy from the body and its environment to power the system. In particular, solar energy and kinetic energy originating from body movement are important sources the wearable system may rely upon. In addition to placing active electronics below the antenna patch, we now also integrate energy harvesters directly on top of the wearable antenna module [1]. In Fig. 3 we present an aperture-coupled shorted wearable solar patch antenna for communication in the $902-928 \mathrm{MHz}$ UHF band.

\section{MIMO techniques for wearable systems}

In indoor environments, wireless communication systems suffer from multipath fading, which significantly reduces the reliability of the data link. In case of 
wearable SFIT units, their deployment in the direct neighborhood of the human body results in important additional shadowing effects. To overcome shadowing and fading, typically higher transmit powers are required to achieve acceptable Bit Error Rates (BERs), which seriously reduces the autonomy of the wearable system. However, by making use of a garment as a platform for the integration of multiple wearable transmit/receive units and by relying on MIMO diversity schemes [8], highly reliable links may be implemented using low transmit power. Fig. 3 demonstrates the improvement in terms of BER for different MIMO schemes that were implemented using a wearable system consisting of two dual-polarized textile antennas integrated in a professional garment. The link quality was studied for a person walking around in an indoor office environment in non-line-of-sight conditions. It is, however, important to approach BER results based on measured instantaneous signal levels like the ones obtained in Fig. 3 with care. In reality, the BER for the real data demodulation suffers from imperfect channel estimation in case of low signal levels. In case of erroneous channel estimation, the space-time codes will not offer the full diversity gain expected from theory. A transmission of 100 frames with channel estimation by 300 BPSK pilot symbols at an average SNR of $10 \mathrm{~dB}$ resulted in a BER at the receiver of $1.7 \mathrm{e}-2$ for a $4 \times 1,8 \mathrm{e}-3$ for a $4 \times 2$ and $1.7 \mathrm{e}-3$ for a $4 \times 4$ link. Clearly, the measured improvements in wireless link quality are found to be much lower than expected from theory. It was found that, by just increasing the average SNR from $10 \mathrm{~dB}$ to $12 \mathrm{~dB}$, for the set of 100 transmitted frames the number of bit errors dropped to zero for the $4 \times 4$ communication link.

\section{Energy-efficient multi-antenna processing techniques}

Although space-time coding (STC) via multiple antenna offers large potential benefits in terms of link quality, it comes at a cost in terms of required processing power to manipulate the codes and to perform accurate channel estimates. Therefore, we have investigated an alternative approach where simple static beamforming is performed used a four-element textile antenna array that transmits a beam confined in the vertical direction [7], resulting in a larger received signal level and reducing the number of received multipath components. This in turn decreases the fluctuations in received signal level. In Fig. 4 we compare space-time coding and static beam forming in non-line-of-sight conditions in an indoor environment. Moreover, BER versus SNR curves are presented for two receiver configurations: a single-antenna receiver without diversity versus a fourantenna receiver implementing maximum ratio combining (MRC). The curves were normalized to include TX array gain, which corresponds to ensuring an equal total transmitted energy per information bit $E_{b, t r}$ at the transmitter. In absence of receiver diversity, the space-time code clearly outperforms beamforming as for the higher $E_{b} / N_{0}$ values, the transmit diversity gain rapidly exceeds the beamforming power gain. In case of receiver diversity, however, the beamforming BER is lower than the BER for space-time coding over the full range we considered. 


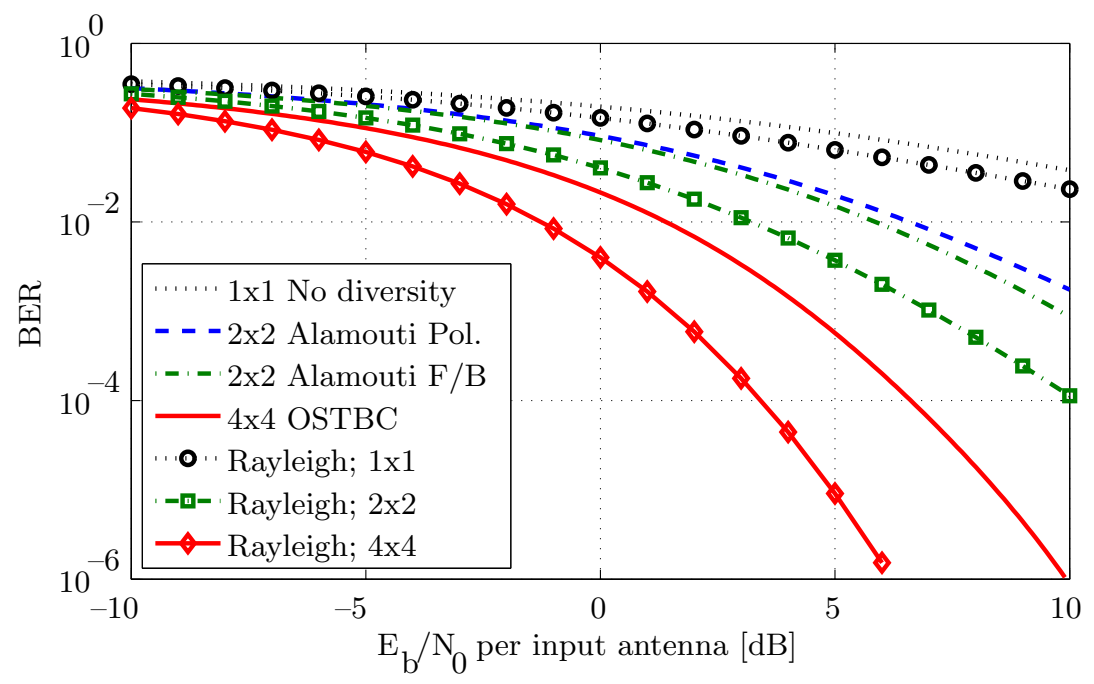

Fig. 3. Bit Error Rate versus Signal to Noise Ratio for various orders of MIMO systems implementing front/back (F/B) and/or polarization (Pol.) diversity by means of Orthogonal Space-Time Block Codes (OSTBCs). The curves derived from measured instantaneous signal levels for a person walking in an indoor environment are compared to the theoretically achievable gains in case of ideal Rayleigh fading.

If we prefer the benefits of additional diversity gain offered by the space-time codes, we need to implement techniques that alleviate the additional processing cost. One approach consists of reducing the overhead in terms of pilot symbols that are needed to make accurate channel estimates at the receiver. This is done by implementing data driven channel tracking [9], where we use the detected data symbols as substitutes for the pilot symbols to keep track in time of the correct channel state. Fig. 5 compares the performance in terms of BER of a $4 \times 4$ MIMO system that has perfect channel knowledge at the receiver with an implementation that makes abundant use of pilot symbols for channel estimation (300 pilot symbols transmitted in separate time-slots for each transmit channel in addition to 528 symbols of space-time coded data per frame) and a link for which data driven channel tracking is implemented at the receiver. We observe that, once the BER drops below 2e-4, the STBC-MIMO system with channel tracking offers a performance similar to that of the MIMO system using large amounts of pilot symbols for channel estimation.

\section{Conclusion}

It was shown that garments of patient and caregiver may be used as platforms to deploy wearable multi-antenna systems that sense the wearer and his/her surroundings. Direct integration of active electronics and energy scavengers onto 


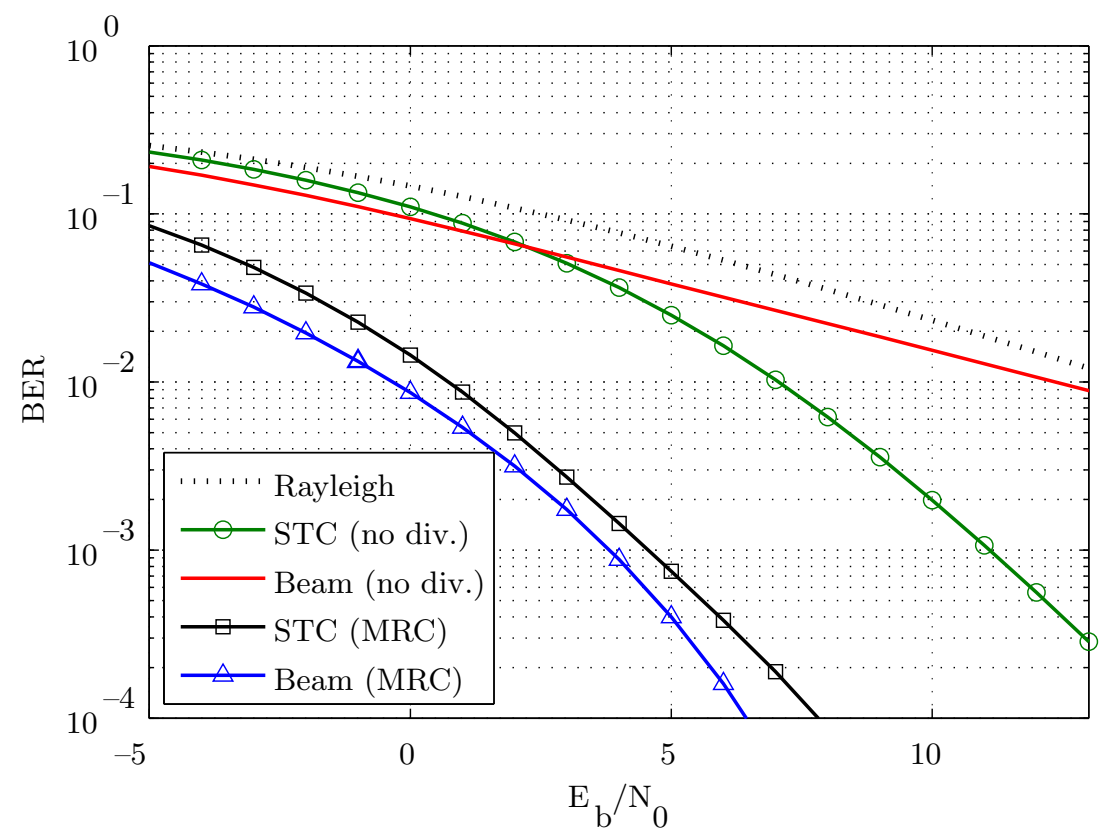

Fig. 4. Bit Error Rate versus Signal to Noise Ratio: comparison between Space-Time Coding (STC) and beam forming (Beam) with $4^{\text {th }}$ order receive diversity (MRC combining) and in absence of diversity.

the active textile antennas lead to compact and energy-efficient modules that are light-weight and comfortable to wear.

\section{References}

[1] Declercq, F., Georgiadis, A., Rogier, H.: Wearable Aperture-Coupled Shorted Solar-Patch Antenna for Remote Tracking and Monitoring Applications. In: Fifth European Conference on Antennas and Propagation - EuCAP 2011. pp. 2992-2996. Rome, Italy (Apr 2011)

[2] Declercq, F., Rogier, H.: Active Integrated Wearable Textile Antenna With Optimized Noise Characteristics. IEEE Trans. on Antennas and Propagation 58(9), 3050-3054 (Sep 2010)

[3] Hertleer, C., Rogier, H., Vallozzi, L., Van Langenhove, L.: A textile antenna for off-body communication integrated into protective clothing for firefighters. IEEE Trans. on Antennas and Propagation 57(4), 919-925 (April 2009)

[4] Locher, I., Klemm, M., Kirstein, T., Troster, G.: Design and characterization of purely textile patch antennas. IEEE Transactions on Advanced Packaging 29(4), 777-788 (December 2006) 


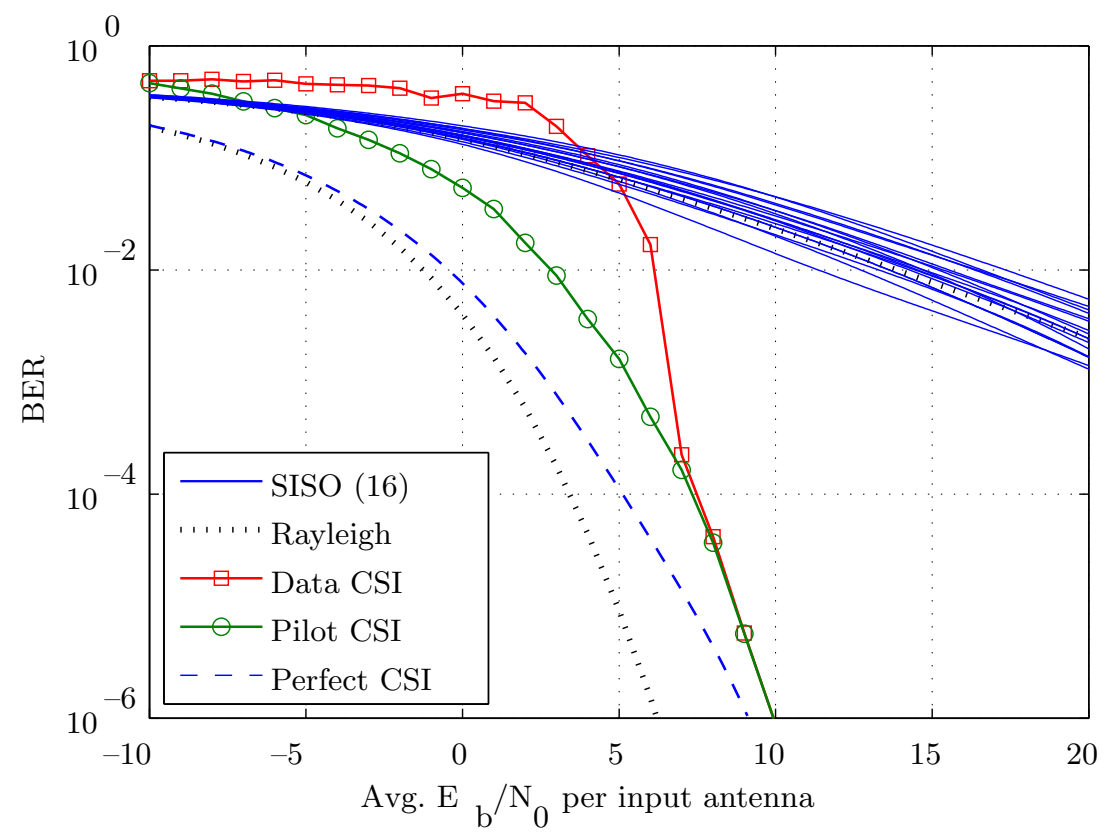

Fig. 5. Bit Error Rate versus Signal to Noise Ratio for the $4 \times 4$ MIMO channel: comparison between perfect channel knowledge, estimation based on pilot symbols and data driven channel tracking.

[5] Salonen, P., Rahmat-Samii, Y.: Textile antennas: Effects of antenna bending on input matching and impedance bandwidth. IEEE Aerospace and Electronic Systems Magazine 22(12), 18-22 (December 2007)

[6] Tronquo, A., Rogier, H., Hertleer, C., Van Langenhove, L.: Robust planar textile antenna for wireless body LANs operating in $2.45 \mathrm{GHz}$ ISM band. IEE Electronic Letters 42(3), 142-146 (2006)

[7] Van Torre, P., Scarpello, M.L., Vallozzi, L., Rogier, H., Moeneclaey, M., Vande Ginste, D., Verhaevert, J.: Indoor Off-Body Wireless Communication: Static Beamforming versus Space-Time Coding. Intern. Journal of Antennas and Propagation (2012)

[8] Van Torre, P., Vallozzi, L., Hertleer, C., Rogier, H., Moeneclaey, M., Verhaevert, J.: Indoor Off-Body Wireless MIMO Communication With Dual Polarized Textile Antennas. IEEE Trans. on Antennas and Propagation 59(2), 631-642 (Feb 2011)

[9] Van Torre, P., Vallozzi, L., Rogier, H., Moeneclaey, M., Verhaevert, J.: Channel Characterization and Robust Tracking for Diversity Reception over TimeVariant Off-Body Wireless Communication Channels. EURASIP Journ. on Advances in Signal Processing (2010) 